\title{
Hepatic lesion radiotherapy: A new option?
}

\author{
Michael Lock MD CCFP FRCPC ${ }^{1}$, Paul C Adams MD², Editor-in-Chief
}

$\mathrm{D}_{\mathrm{R}}^{\mathrm{r}}$ Michael Lock is the Director and Head of the Division of Radiation Oncology for the London Health Sciences Centre and an assistant professor at the University of Western Ontario in London, Ontario. He is involved with the development of image-guided and radiobiologically guided radiotherapy for the London Regional Cancer Program.

PA: We often believe that radiation therapy is not ideal for liver tumours.

ML: Historically, radiation was not used for hepatic lesions because radiation to the normal liver can result in significant hepatic toxicity. The dose needed to damage the tumour far exceeds the amount that would result in hepatic failure. However, with the development of new technologies and techniques, we are able to focus the radiation more precisely on the lesion; decrease the dose to normal structures by using methods such as image guidance to account for organ motion; and individualize the dose regimen based on the patient's radiobiological characteristics. The first major technological improvement has been the development of three-dimensional conformal radiotherapy as opposed to conventional irradiation. This has enabled us to 'sculpt' the dose away from normal tissues and to provide a higher dose to the tumour. The second advancement in technology has been the development of daily image guidance. The motion of the liver

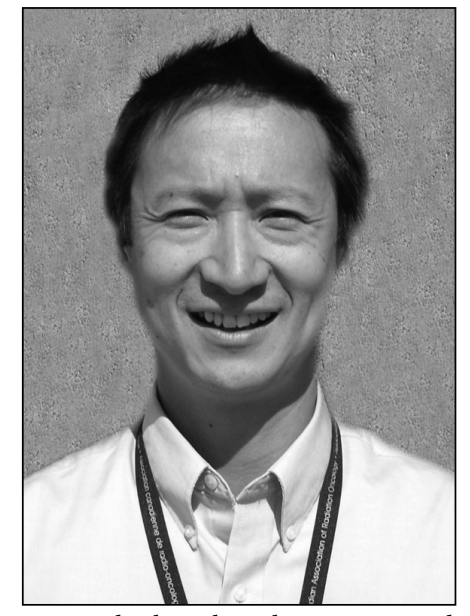

Dr Michael Lock is the Director and Head of the Division of Radiation Oncology for the London Health Sciences Centre and an assistant professor at the University of Western Ontario in London, Ontario. He is involved with the development of image-guided and radiobiologically guided radiotherapy for the London Regional Cancer Program provide the maximum dose to each patient as opposed to a standard dose given to an average patient.

PA: Who is the ideal candidate for conformal radiation therapy to a liver lesion?

ML: In terms of the type of tumour, the largest body of literature is in primary hepatocellular carcinoma and metastatic colorectal carcinoma. However, there is increasing experience with all metastatic lesions including cholangiocarcinoma, breast carcinoma and pancreatic carcinoma. In terms of technical issues, patients must be able to lie still on their back for approximately $10 \mathrm{~min}$ and have no contraindications to radiotherapy (eg, previous irradiation to the region of interest). One of the most important parameters is the amount of normal liver remaining. Retrospective data indicate that high doses of radiation therapy can be delivered without significant toxicity, as long as a certain amount of normal liver is spared. The Colorado Multiple Institutional Review Board (1) and the Princess Margaret Hospital protocol (2) have provided parameters to select patients for treatment. Specifically, Colorado's constraint is that $700 \mathrm{~mL}$ of the liver is spared more than $15 \mathrm{~Gy}$ of the dose and the Princess Margaret Hospital protocol constraint is that $800 \mathrm{~mL}$ of the liver is uninvolved. Additional methods to determine the risk of liver toxicity, based on radiobiological data that account for factors such as variation in the dose intensity across the noration of a larger region where the tumour may be located during treatment. If we can localize the tumour in four dimensions, then we can reduce the size of the irradiated field and, therefore, reduce the amount of normal tissue irradiated. A simple example of image guidance is the use of infrared markers placed on a patient's chest to track the respiratory motion. We can project where the liver lesion is anticipated to be during certain parts of the respiratory cycle. Therefore, we only turn on the beam during this portion of the cycle. This can reduce the volume of normal tissue irradiated. Finally, algorithms are now available that allow our physicists and radiobiologists to calculate the risk of causing damage to the liver at different doses and regimens. This is unique to each patient due to variations in their anatomy, liver motion, type of tumour and comorbidities. These algorithms allow us to mal liver, further improve our ability to maximize dose and minimize toxicity. The ideal candidate would also have a single lesion without active systemic disease. However, because these patients are best treated by surgery, radiation oncologists see patients with single lesions only if the patient is inoperable due to some comorbid contraindication to surgery or anatomical contraindication such as involvement of major vessels and/or multiple lobes. In terms of patient-related factors, comorbidities such as cirrhosis and hepatitis can increase the risk of radiationinduced liver disease and are relative contraindications. In other words, the ideal candidate is an otherwise healthy patient with an unresectable hepatocellular carcinoma or metastatic colorectal carcinoma, with more than $800 \mathrm{~mL}$ of normal liver and no previous upper abdominal radiation history.

${ }^{1}$ London Regional Cancer Centre, London, Ontario; ${ }^{2}$ University Hospital, London, Ontario

Correspondence: Dr Michael Lock, London Regional Cancer Centre, 790 Commissioners Road East, London, Ontario N6A 4 L6.

Telephone 519-685-8600 ext 53347, fax 519-685-8736, e-mail michael.lock@lhsc.on.ca

Received and accepted for publication January 25, 2008 

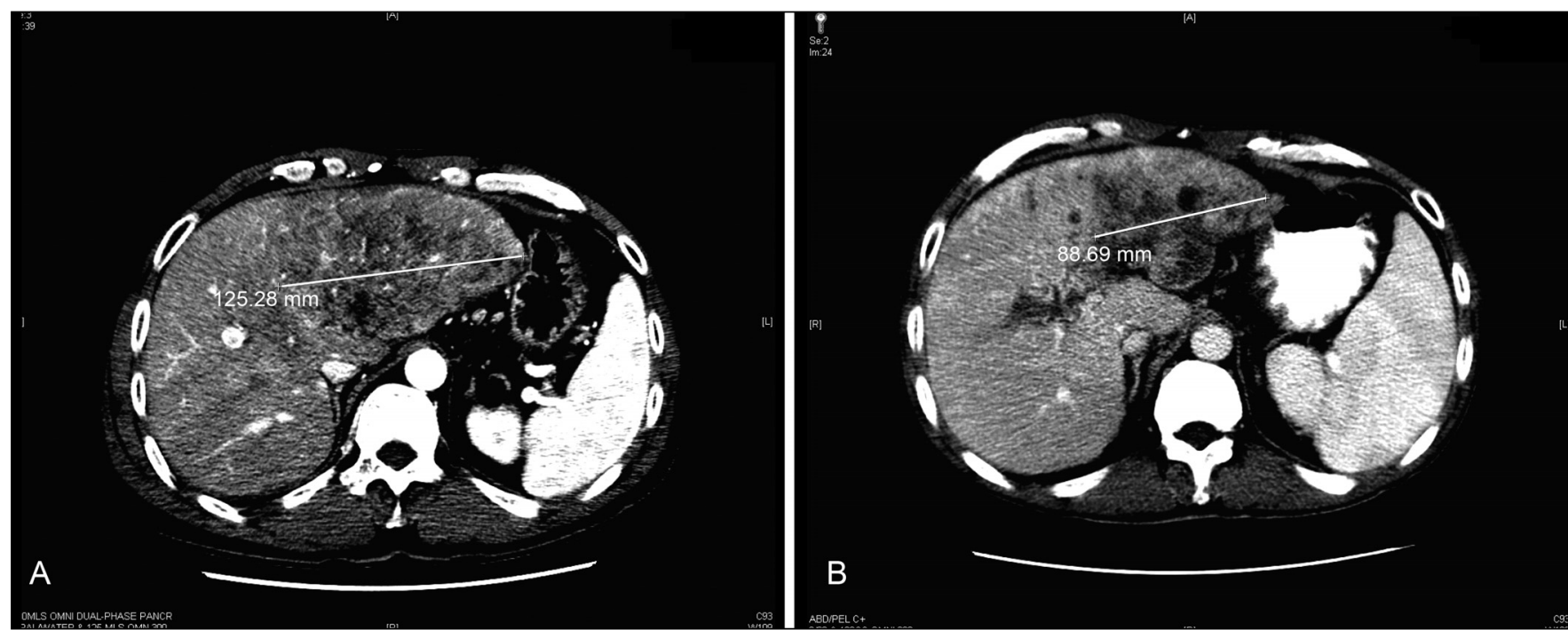

Figure 1) A Pretreatment computed tomography of the liver of a 50-year-old man with hepatocellular carcinoma. B Computed tomography of the liver three months after radiation therapy

PA: Can you describe the treatment from the patient perspective?

ML: The patient goes through a two-step assessment process after the consultation. First, the patient is simulated using a respiratory-gated, four-dimensional computed tomography scan. The normal tissues and liver lesion are contoured. Then, a dosimetric plan is developed that details how the radiation will be delivered. This step includes the selection of the method of daily image guidance that will best localize the tumour during treatment. Second, a radiobiological algorithm is used to calculate the risk of toxicity using different regimens. A regimen that maximizes the dose, but that does not allow the toxicity to exceed 5\%, is chosen. Different centres use different toxicity thresholds to exclude patients. Some patients cannot receive a significant dose without exceeding the 5\% level, and are excluded. This can be a difficult conversation because many of these patients are not eligible for, or have failed, all other therapies. Some patients receive a low dose that is simply given palliatively to retard the growth of the tumour. Once deemed eligible, the patient begins radiation treatment over a period determined by the radiobiological calculations. This ranges from one to six weeks. Each treatment lasts longer than a standard radiation treatment because image guidance requires increased treatment times. This may last approximately $10 \mathrm{~min}$ (Figure 1 ).

PA: What are the challenges for the radiation oncologist?

ML: Radiation oncology has seen the development of new technology that offers our patients significant improvements in tumour cell kill and, at the same time, reductions in toxicity. However, these technologies necessitate new and expensive equipment. Staff at multiple levels need to gain new skills and experience in management that were not part of their initial education. The principles of individualized dose regimen selection, image guidance and radiobiological guidance are comparatively new concepts to radiation oncology. The knowledgeable application of these principles is critical for hepatic irradiation. In addition, to plan and treat using these techniques can be time consuming. In the age of waiting lists and cost reductions, the time and skill development required, and the expense of this treatment are significant challenges. Due to the need to have a facility with fourdimensional computed tomography, image-guided radiotherapy equipment and radiobiological expertise, this technique is only offered in a limited number of centres. Treatment should continue to be provided in clinical trials or with considerable quality assurance oversight.

In terms of technical challenges, the radiation oncology community is only starting to delve into this area. Many basic management parameters have been identified; for example, data from Michigan (3) have indicated that higher doses are better than lower doses. However, exactly how high the dose should be for each treatment, how many fractions in total are optimal and how much time should pass between treatments are still to be resolved. Therefore, there are many fundamental technical issues to be clarified. Developing proficiency in the new principles of image guidance and radiobiological dose selection in many centres will allow multicentre trials to be initiated and the technical issues to be resolved.

PA: How would you compare conformal radiation therapy with other ablation treatments such as radiofrequency ablation, ethanol ablation, chemoembolization or radioactive beads?

ML: No head-to-head studies comparing these modalities have been published. Radiation represents an attractive alternative because it is noninvasive. In addition, radiation is not limited by some of the anatomical issues associated with other therapies. For example, radiation is less likely to be hindered by the size, multiplicity and location (eg, near vessels) of liver lesions. Outcome data for radiation continue to accumulate, but are primarily case series data. This level of evidence limits our ability to compare outcomes and interpret results. Unfortunately, no phase III data have been published because the techniques and regimens are still being developed. The best data are from Dr Laura Dawson, who pioneered the use of radiation for hepatic lesions. Case series data published by Dawson et al (3) have shown median survivals of 11 to 15 months with the use of radiation in unresectable hepatobiliary cancer, which compares favourably with other modalities (4). 
The side effects of this treatment also differ. The use of radiation is sometimes associated with fatigue and abdominal discomfort. The major concerns are fortunately uncommon. The primary concern is radiation-induced liver disease. This is essentially liver failure with a constellation of signs and symptoms that occur within three months of radiation therapy, including elevated liver markers, hepatomegaly and ascites. Other major concerns include kidney damage, gastric ulcers, bleeding and bowel obstruction.

PA: Is there a role for conformal radiation therapy in combination with other therapies?

ML: From a technical ability-to-treat point of view, the use of high-precision external beam radiotherapy can be complementary or an alternative to other treatments. For example, radiation may be offered to patients with a large tumour that exceeds the size that can be treated by radiofrequency ablation. Possibilities include the shrinkage of these lesions to enable radiofrequency ablation. A lesion that is treated by chemoembolization may be found to have an alternate vascular supply that cannot be occluded. Radiation can play a complementary role in these cases and be added to this modality. One study (5) demonstrated that in patients who had failed transarterial chemoembolization, local radiation induced a substantial $67 \%$ additional tumour response. Future studies will surely investigate the use of a standard sequence of these treatments to maximize tumour ablation. Furthermore, radiation is a very local treatment. Postradiation failures are commonly within the liver. Data from Asia (6) have demonstrated that the use of transarterial chemoembolization is essential in improving the outcome of patients by reducing out-of-field failures. These data suggest that radiation should be used as a component in the management of hepatic lesions as opposed to a singular modality. Whether this should be sequential or concomitant is still to be resolved.
PA: Can conformal radiation therapy be used on other tumours in the gastrointestinal tract?

ML: Yes, conformal radiation therapy is becoming a standard and ubiquitous technique. There are already subtypes of conformal radiation, such as intensity-modulated radiotherapy and TomoTherapy (TomoTherapy Incorporated, USA), which offer an even greater ability to deliver high doses to the tumour and spare normal tissues. However, the use of these techniques is still limited by motion and the ability to localize gastrointestinal tract tumours in real time. A technique called stereotactic body radiosurgery is being developed to minimize this limitation. This technique may expand the role of radiation in other tumours of the gastrointestinal tract.

\section{REFERENCES}

1. Schefter TE, Kavanagh BD, Timmerman RD, Cardenes HR, Baron A, Gaspar LE. A phase I trial of stereotactic body radiation therapy (SBRT) for liver metastases. Int J Radiat Oncol Biol Phys 2005;62:1371-8.

2. Tse RV, Hawkins M, Lockwood G, et al. Phase I study of individualized stereotactic body radiotherapy for hepatocellular carcinoma and intrahepatic cholangiocarcinoma. J Clin Oncol 2008;26:657-64.

3. Dawson LA, McGinn C, Normolle D, et al. Escalated focal liver radiation and concurrent hepatic artery fluorodeoxyuridine for unresectable liver malignancies. J Clin Oncol 2000;18:2210-8.

4. Hawkins MA, Dawson LA. Radiation therapy for hepatocellular carcinoma. Cancer 2006;106:1653-63.

5. Seong J, Park HC, Han KH, et al. Local radiotherapy for unresectable hepatocellular carcinoma patients who failed with transcatheter arterial chemoembolization. Int J Radiat Oncol Biol Phys 2000;47:1331-5.

6. Shim SJ, Seong J, Lee IJ, Han KH, Chon CY, Ahn SH. Radiation-induced hepatic toxicity after radiotherapy combined with chemotherapy for hepatocellular carcinoma. Hepatol Res 2007;37:906-13. 


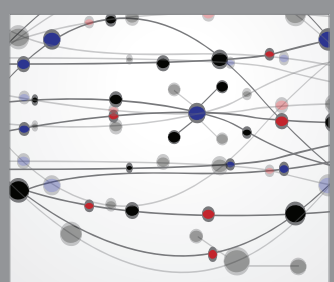

The Scientific World Journal
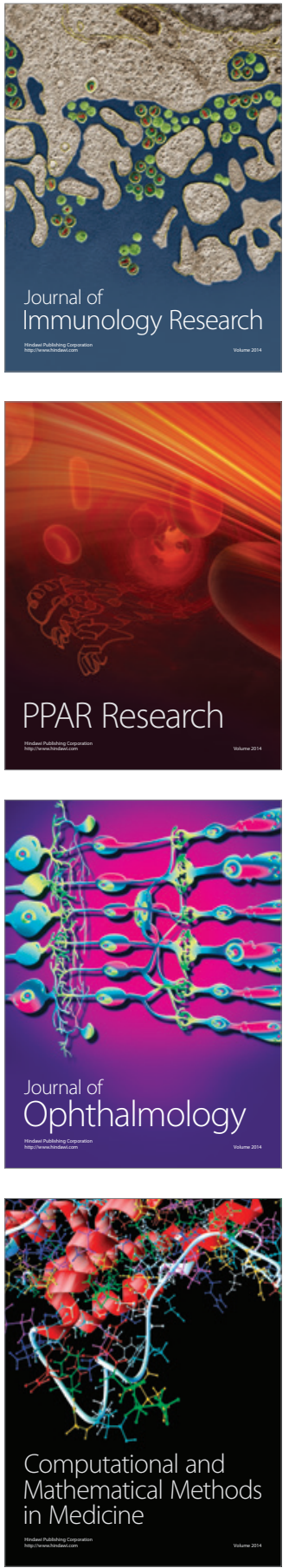

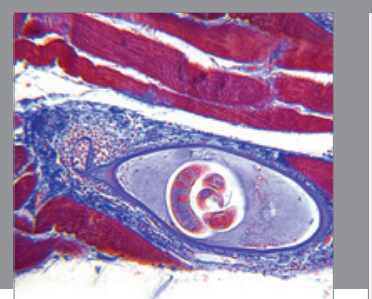

Gastroenterology Research and Practice

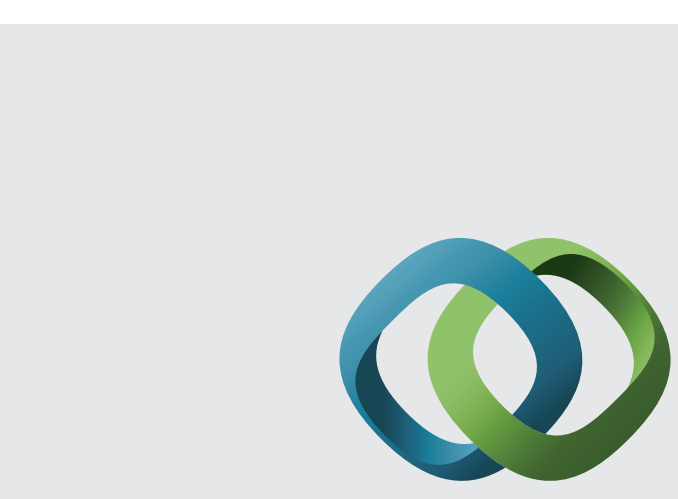

\section{Hindawi}

Submit your manuscripts at

http://www.hindawi.com
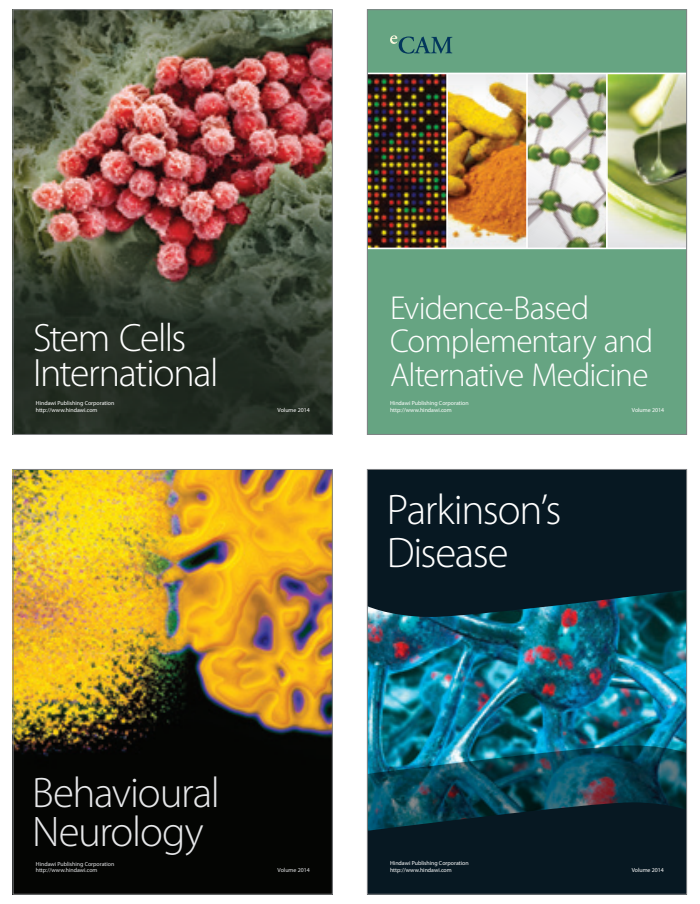
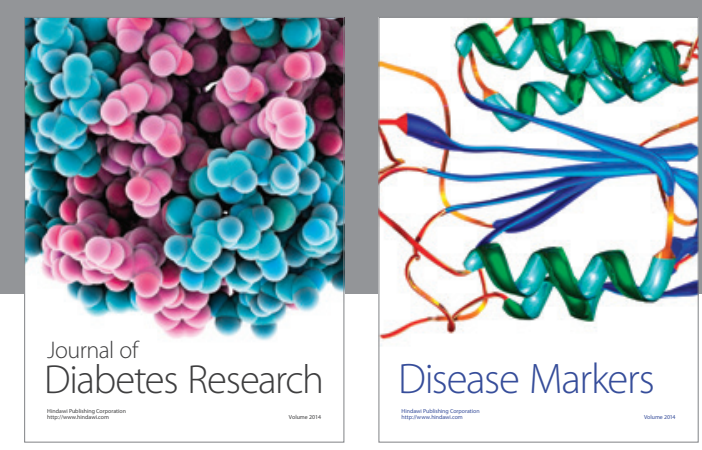

Disease Markers
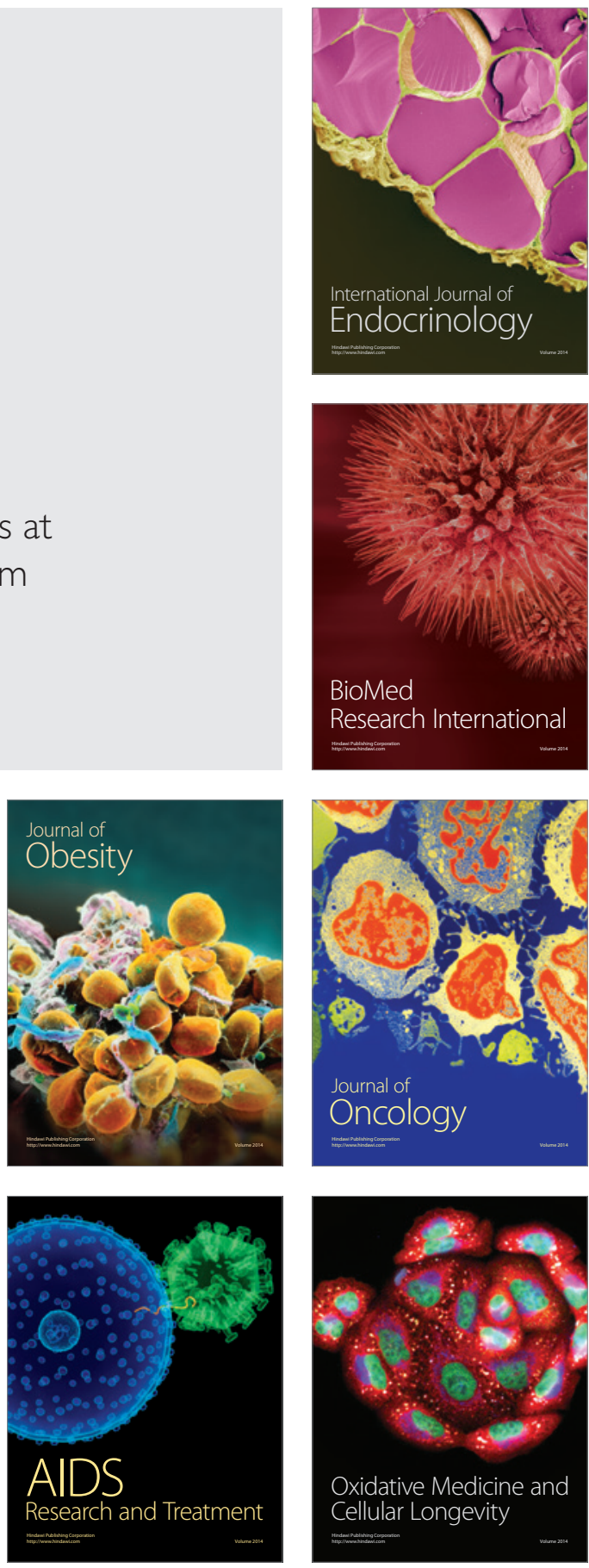